\title{
Surface Roughness Evaluation of Nano Composites with Different Polishing Techniques: (In-Vitro Study)
}

\section{Nehad Wafik Basha*, Mohamed Mahmoud Abdel-Mohsen and Heba Mohamed Taher}

Department of Conservative Dentistry, October University for Modern Sciences and Arts (MSA), Cairo, Egypt

*Corresponding Author: Nehad Wafik Basha, Department of Conservative Dentistry, October University for Modern Sciences and Arts (MSA), Cairo, Egypt.
Received: May 17, 2021

Published: October 15, 2021

(C) All rights are reserved by Nehad Wafik

Basha., et al.

\begin{abstract}
Background and Objective: The purpose of this study was to evaluate the surface roughness of Nano resin composites with different polishing techniques (in-vitro study).

Method: 120 composite specimens with a $6 \mathrm{~mm}$ diameter and a thickness of $3 \mathrm{~mm}$ were fabricated from the nanofilled Filtek Z350 XT (C1) and the nanohybrid Filtek Z250 XT (C2) and equally divided into two main groups ( $\mathrm{n}=60)$, 20 specimens of each composite were polished with PoGo single step micro-polishers (P1), Sof-Lex multiple steps polishing system (P2) and polyester strip was used as the control group (P0), ( $\mathrm{n}=20)$, a profilometer was used to measure the surface roughness value after specimens' polishing (Ra), the data were statistically analyzed using Repeated measures Analysis of Variance (ANOVA) and Bonferroni's post-hoc test (P $\leq 0.05$ ) was used for pair-wise comparisons when ANOVA test is significant.

Results: There was a statistically significant difference between mean Ra of different polishing systems (P-value $=0.001$, Effect size = 0.269), no statistically significant difference between PoGo discs and Sof-Lex discs; both showed statistically significantly higher mean Ra than Polyester strip.

Conclusion: 1. The nanofilled Filtek Z350 XT resin composite is a good choice material for the clinical use. 2. the one-step PoGo micro-polisher produced better surface quality in terms of roughness than the multiple steps Sof-Lex polishing system.
\end{abstract}

Keywords: Resin Composite; Surface Roughness

\section{Introduction}

Beauty and natural tooth appearance of resin composite together with their conservative approach are the main reasons for the increasing demands for its use since their introduction in the late 1950's. Resin composite is a material that is made of an inorganic filler phase and an organic matrix phase with a coupling agent that bonds the filler to the matrix [70].
Composites were traditionally classified depending upon their filler size into macrofilled, microfilled and hybrid [12]. Since the introduction of nanotechnology, a new classification for resin composites had been attempted to be classified as nanofilled and nanohybrids [49]. Nanofilled contains only nanoscale particles (20-75 $\mathrm{nm}$ ) [12], which are made of nearly uniform nanometric particles and create nanoclusters as secondarily formed fillers while nano- 
hybrids are made of particles of various sizes, from the micrometric to nanometric scale [23]. The nanomer and nanocluster filler particles in the nanofill type provide surface smoothness, high polish retain ability and superior gloss as well as adequate mechanical properties [2,12]. The clinical success of composite restorations is related to surface smoothness, thus, finishing and polishing is of paramount importance for the success and longevity of these restorations $[2,29]$.

Increased surface roughness allows accumulation of biofilm leading to the development of gingivitis and discoloration of the restoration [18]. It is dependent on the type of the composite material and polishing system used [17]. Finishing procedure removes scratches created by instruments and provides smooth surface with particle size of more than $25 \mu$ whereas polishing creates an enamel-like luster to the restoration and increases the surface energy of the restoration with particle size lesser than $25 \mu \mathrm{m}$ [2].

Diamond or multi-fluted tungsten carbide burs are used for gross finishing followed by polishing using polishing wheels and discs with flexible or semi flexible abrasives coated with aluminum oxide $\left(\mathrm{Al}_{2} \mathrm{O}_{3}\right)$ abrasive or fine diamond particles. Polishing devices are available including $\mathrm{Al}_{2} \mathrm{O}_{3}$ impregnated Sof-Lex wheels and diamond impregnated rubber PoGo polishing discs, which operate as multi-step and single step polishing systems simultaneously. Multistep polishing system use medium $(40 \mu \mathrm{m})$, fine $(24 \mu \mathrm{m})$ and ultrafine $(8 \mu \mathrm{m})$ grit sequence in while PoGo product rubber diamond polisher uses a single step $[2,20,23,29]$.

Therefore, it seems worthwhile to evaluate the surface roughness of the Nano resin composites with different polishing techniques. The study was carried out under the following regards, Comparing the surface roughness of two different types of resin composite (Filtek Z350 XT nanofilled and Filtek Z250 XT nanohybrid) and comparing the surface roughness of two different types of polishing system (PoGo single step and Sof-Lex multiple steps).

The following hypothesizes were tested:

- There would be no difference in surface roughness values between nanofilled and nanohybrid resin restoratives.

- There would be no difference between the single step and multiple steps polishing systems.

\section{Materials and Methods}

Two widely used commercial resin composites (Table 1) were evaluated in this study. A total of 120 resin composite disks with a thickness of $3 \mathrm{~mm}$ and $6 \mathrm{~mm}$ in diameter were fabricated using a special cylindrical split Teflon mold in order to standardize specimens' dimension. The composite was inserted into the mold in two incremental layers using OptraSculpt (Ivoclar Vivadent, Schaan, Liechtenstein) modeling instrument. Teflon mold contained uncured composite was covered on both sides with a transparent polyester strip then held between two microscopic glass slides gently pressed together to remove excess material. An axial load of $500 \mathrm{~g}$ was applied on the top of microscopic glass slide for $20 \mathrm{sec}$ to promote smoothness, removing voids and extrude any excess of material [44]. Using light emitting diode (Elipar curing unite, $3 \mathrm{~m}$ ESPE St. Paul, USA (wavelength: 430 - 480 nm, light intensity: 1200 $\mathrm{mW} / \mathrm{cm}^{2}$ ), (LED) light curing unit, the first inserted resin composite increment was light-cured for $20 \mathrm{sec}$ following manufacturer instructions. Then, the second inserted resin composite increment had been light-cured for $20 \mathrm{sec}$ through the polyester matrix strip and the microscopic glass slide. Additional $20 \mathrm{sec}$ light-curing on both sides of the composite disc specimen were done after removing the polyester matrix strips and the microscopic glass slides to insure proper curing. The light intensity of the curing unit was varified regularly every 5 exposures by means of a digital dental radiometer (Ivoclar Vivadent, Schaan, Liechtenstein). Standardization of the distance between light source and specimen was obtained by the thickness of the glass slide ( $1 \mathrm{~mm}$ ) and polyester matrix strip, which gave smooth surfaces for the composite specimens [1]. After light-curing, the cured composite specimens were then removed from the Teflon mold and checked by using hand held magnifying glass lens (Jinhua Top Optical Instrument Co., Ltd, China) X5 for detecting any surface defect, crack or/and air bubbles, which if present the specimen was discarded. The non-irradiated surface of the cured composite specimen was marked. Then, the cured composite specimen was picked up by tweezer, which was applied to the sides of the cylinder to protect the flat polyester-formed surface of the cured composite specimen from any damage or contamination.

The cured composite specimens were inserted into the second Teflon mold (Figure 1) of $2 \mathrm{~mm}$ thickness making it possible to polish the cured composite specimens on a tight-fixed base. Thus, the residual thickness can be controlled [23]. The cured composite 


\begin{tabular}{|c|c|c|c|}
\hline Brand name & Specification & Composition & $\begin{array}{c}\text { Manufacturer } \\
\text { (Batch No.) }\end{array}$ \\
\hline $\begin{array}{l}\text { Filtek }^{\mathrm{TM}} \mathrm{Z} 350 \mathrm{XT} \\
\text { Universal Restorative } \\
\text { Body } \\
\text { Shade: A2 }\end{array}$ & Nanofilled composite & $\begin{array}{c}\text { Matrix: Bis-GMA }{ }^{1}, \mathrm{UDMA}^{2} \text {, TEGDMA } \\
\text { resins. }\end{array}$ & $\begin{array}{c}\text { 3M ESPE, St. Paul, } \\
\text { MN, USA } \\
\text { (NA44144) }\end{array}$ \\
\hline $\begin{array}{l}\text { Filtek }^{\mathrm{TM}} \mathrm{Z} 250 \mathrm{XT} \\
\text { Universal Restorative } \\
\text { Shade: A2 }\end{array}$ & Nanohybrid composite & $\begin{array}{l}\text { Matrix: Bis-GMA, UDMA, Bis- EMA, PEGDMA and TEGDMA resins. } \\
\text { Fillers: Combination of surface modified zirconia/silica. The inor- } \\
\text { ganic filler loading is } 81.8 \% \text { by weight ( } 67.8 \% \text { by volume) with a } \\
\text { particle size of } 20 \mathrm{~nm} \text { for the silica and approximately } 0.1-10 \mu \mathrm{m} \\
\text { for the zirconia/silica. } \\
\text { Filler loading: } 67.8 \% \text { by volume. }\end{array}$ & $\begin{array}{c}\text { 3M ESPE, St. Paul, } \\
\text { MN, USA } \\
\text { (NA60349) }\end{array}$ \\
\hline
\end{tabular}

Table 1: Brand name, specification, composition and manufacturer of resin composite.

${ }^{1}$ Bisphenol-A-glycol-dimethacrylate.

${ }^{2}$ Urethane-dimethacrylate.

${ }^{3}$ Tri-ethylene-glycol-dimethacrylate.

${ }^{4}$ Polyethylene glycol dimethacrylate.

${ }^{5}$ Bisphenol-A-polyethylene-glycol-diether-dimethacrylate.

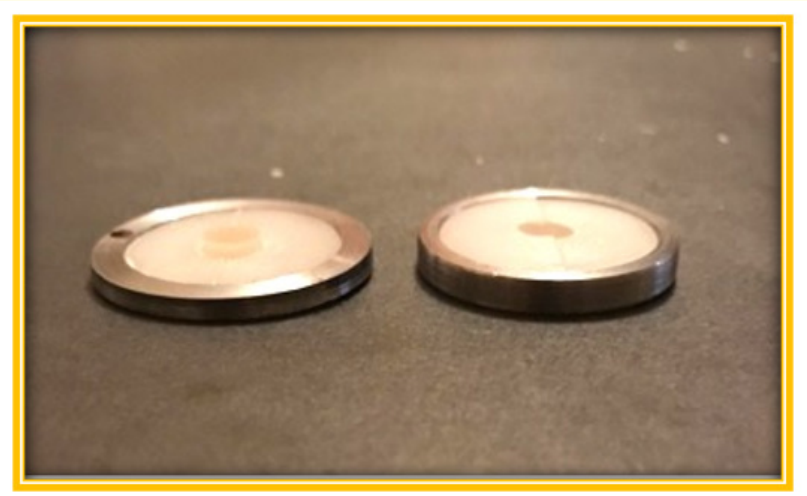

Figure 1: The specimens were inserted into the second Teflon mold, marked on the side to facilitate the polishing procedures. surfaces had been marked from their side to ensure that all polishing took place in the same direction i.e., parallel to the surface [20]. According to manufacturer instructions, the cured composite specimens were polished with three different polishing techniques (Table 2), ( $\mathrm{n}=20$ ) the first group (P1) the pogo single step micro polishers, The PoGo polishers were manufactured in different shapes including cups, points or discs. In this study, disc shape polishers were used in order to obtain a direct contact with the surfaces of specimens [31]. Although the manufacturer recommends pretreatment with Enhance system to obtain favorable results, some investigators have used this system as a one-step method without any pre-treatment and reported no beneficial results on the surface quality with the pre-treatment step $[22,66]$. Therefore, PoGo was used as a one-step method in the present study. 


\begin{tabular}{|c|c|c|c|c|}
\hline \multicolumn{2}{|c|}{ Material } & $\begin{array}{c}\text { Product } \\
\text { Specifications }\end{array}$ & Composition & $\begin{array}{c}\text { Manufac- } \\
\text { turer (Batch } \\
\text { No.) }\end{array}$ \\
\hline \multicolumn{2}{|c|}{$\begin{array}{l}\text { Transparent } \\
\text { Polyester strip }\end{array}$} & $\begin{array}{l}\text { Transparent } \\
\text { rectangle- } \\
\text { shaped univer- } \\
\text { sal strip. }\end{array}$ & $\begin{array}{c}\text { Flexible polyester } \\
\text { used for contour- } \\
\text { ing and polishing } \\
\text { of resin compos- } \\
\text { ite restorations } \\
\text { (length: } 100 \mathrm{~mm} \text {, } \\
\text { width: } 8 \mathrm{~mm} \text {, } \\
\text { thickness: } 0.05 \\
\text { mm). }\end{array}$ & $\begin{array}{c}\text { Kerr-Hawe, } \\
\text { Bioggio, } \\
\text { Switzerland } \\
\text { (40611) }\end{array}$ \\
\hline \multicolumn{2}{|c|}{$\begin{array}{l}\text { PoGo discs } \\
\text { micro-polishers } \\
\text { (One step) }\end{array}$} & $\begin{array}{l}\text { Pre-mounted, } \\
\text { single use } \\
\text { diamond } \\
\text { impregnated } \\
\text { cured urethane } \\
\text { dimethacrylate } \\
\text { resin polishing } \\
\text { devices. }\end{array}$ & $\begin{array}{c}\text { Polymerized } \\
\text { urethane dimeth- } \\
\text { acylate resin, fine } \\
\text { diamond powder, } \\
\text { silicon oxide, } \\
\text { plastic latch-type } \\
\text { mandrel. }\end{array}$ & \begin{tabular}{|} 
Dentsply \\
Caulk, \\
Milford, \\
Delaware, \\
USA \\
$(662010 Y)$
\end{tabular} \\
\hline \multirow{3}{*}{$\begin{array}{l}\text { Sof-Lex } \\
\text { polish- } \\
\text { ing } \\
\text { system } \\
\text { (Multi- } \\
\text { step) }\end{array}$} & $\begin{array}{l}\text { Sof-Lex } \\
\text { extra- } \\
\text { thin } \\
\text { discs }\end{array}$ & $\begin{array}{c}\text { Medium-grit } \\
\text { flexible discs for } \\
\text { contouring and } \\
\text { polishing. }\end{array}$ & $\begin{array}{c}\text { Aluminum oxide } \\
\text { extra thin flexible } \\
\text { disc (diameter: } \\
12.7 \text { mm, thick- } \\
\text { ness: } 0.5 \text { inches). }\end{array}$ & $\begin{array}{c}\text { 3M ESPE, } \\
\text { St. Paul, MN, } \\
\text { USA } \\
(4673856)\end{array}$ \\
\hline & \multirow{2}{*}{$\begin{array}{l}\text { Sof-Lex } \\
\text { spiral } \\
\text { wheels }\end{array}$} & $\begin{array}{c}\text { Fine grit (beige) } \\
\text { pre-polishing } \\
\text { spiral wheel. }\end{array}$ & $\begin{array}{c}\text { Aluminum oxide } \\
\text { impregnated in } \\
\text { a thermoplastic } \\
\text { elastomer (diam- } \\
\text { eter: } 12.7 \mathrm{~mm} \text {, } \\
\text { thickness: } 0.13 \\
\text { inches). }\end{array}$ & $\begin{array}{c}\text { 3M ESPE, } \\
\text { St. Paul, MN, } \\
\text { USA } \\
\text { (NA46599) }\end{array}$ \\
\hline & & $\begin{array}{l}\text { Ultra fine grit } \\
\text { (pink) for } \\
\text { final polishing. }\end{array}$ & $\begin{array}{c}\text { Aluminum oxide } \\
\text { impregnated in } \\
\text { a thermoplastic } \\
\text { elastomer (diam- } \\
\text { eter: } 12.7 \mathrm{~mm} \text {, } \\
\text { thickness: } 0.13 \\
\text { inches). }\end{array}$ & $\begin{array}{c}\text { 3M ESPE, } \\
\text { St. Paul, MN, } \\
\text { USA } \\
\text { (NA46599) }\end{array}$ \\
\hline
\end{tabular}

Table 2: Specification, composition and manufacturer of polishing system.

The flat broad surface of the one step PoGo polishing system that uses diamond micro-polisher disc were used (Figure 2). These discs are indicated for single use. Thus, one disc was used for each

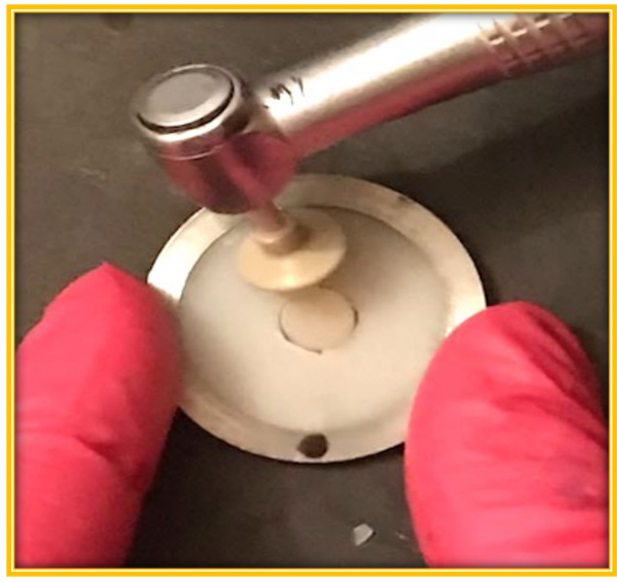

Figure 2: Polishing the specimen with PoGo micro-polisher disc.

specimen. The discs were attached to a slow-speed micro-motor Micromotor handpiece; Strong, South Korea (20.000 - 40.000rpm) handpiece NSK Ti-Max Electric Handpiece, Japan. The speed was adjusted at 10,000 rpm for the initial $15 \mathrm{sec}$ followed by $15 \mathrm{sec}$ at 20,000 rpm for each specimen [27].

Unlike to any other rotary instrument, the PoGo polishers are designed for use without water, which may generate heat with prolonged contact, thus, they were first applied with a light and intermittent pressure in buffing motion to increase the surface luster.

In the Sof-Lex system, the manufacturer strictly recommends the use of Sof-Lex extra thin disc prior to the use of spirals in accordance with [41], who found the use of Sof-Lex extra thin disc prior to the use of Sof-Lex spirals had produced smoother surface than using spirals alone, therefore, The second group of the cured composite specimens were polished with Sof-Lex contouring and polishing system (P2) following the protocol recommended by the manufacturer; medium grit Sof-Lex extra thin contouring disc (orange) was first used followed by the Sof-Lex spiral wheels fine grit (beige) pre polishing disc and ultra fine polishing disc (pink)for the final surface luster.

For optimum results, the spiral wheels were used on wet surfaces with moderate pressure. The spiral wheels were immersed in 
water before polishing the specimens as well as the surface of the specimens had been wetted with a microbrush. The specimen was rinsed and dried by a microbrush between the successive discs in the same specimen in order to eliminate any created debris from instruments side [41].

Abrasive Sof-Lex disc in the kit was attached by a metal hub to an autoclavable metal mandrel. The specimens were contoured and polished with medium, fine, and ultra fine discs by using linear movements with a slow-speed micro-motor handpiece (Figure 3-5). For each resin composite specimen, the medium grit discs were used for a gross contouring at medium speed of 10,000 rpm for $15 \mathrm{sec}$ without water spray then the specimens were rinsed to remove any material's powder and dried with microbrush while the fine grit Sof-Lex and the ultra-fine grit spiral wheels were used at a high speed of 20,000 rpm for $15 \mathrm{sec}$ [10].

The Sof-Lex extra thin discs are single use, thus, a new polishing disc was used for each specimen and was discarded after each use. On the other hand, the Sof-Lex spiral wheels are multiuse, thus, they were replaced each five specimens [23]. The third group (P0) were left without surface treatment, just curing under the polyester strip and were used as a control group. Another parameter of importance was the standardization of the force applied on the surface during polishing. In the present study, to minimize the varia-

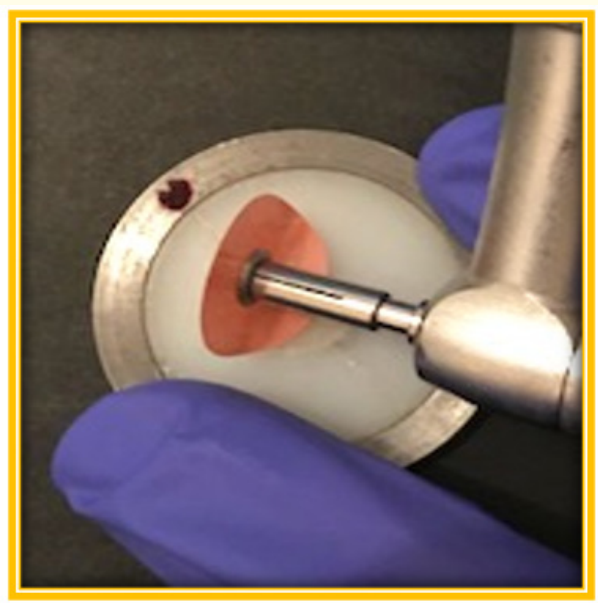

Figure 3: Contouring the specimen with a Sof-Lex extra-thin disc (Orange).

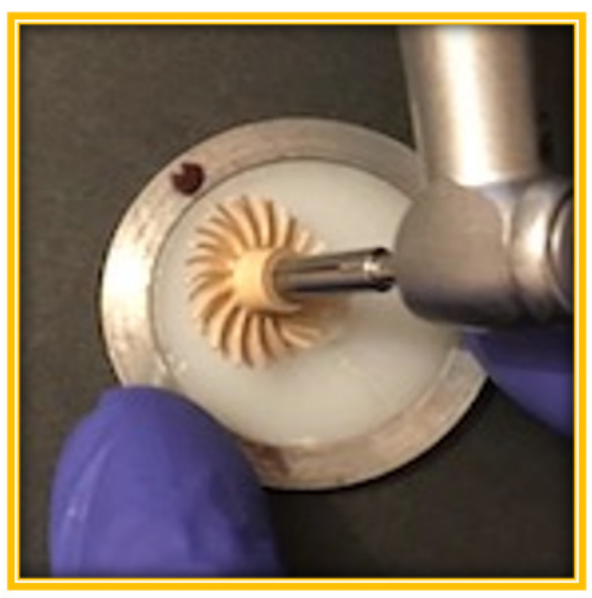

Figure 4: Pre-polishing the specimen with the Sof-Lex spiral wheel fine (beige).

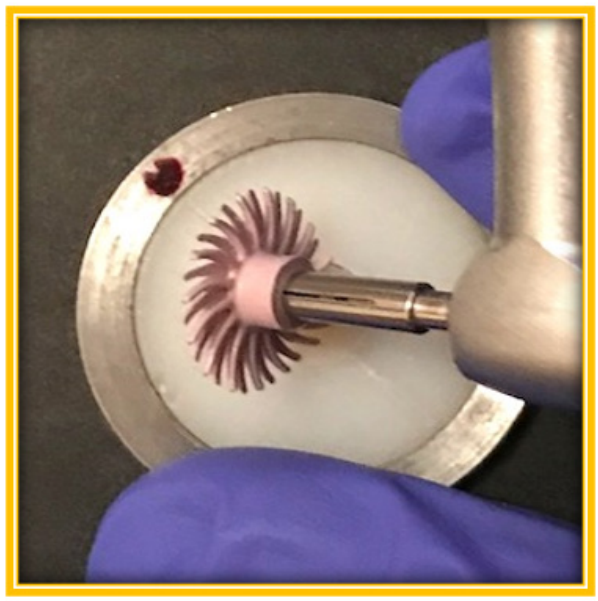

Figure 5: Polishing the specimen with the Sof-Lex ultra fine (Pink).

tion in the force applied, single operator used a light force with a constant movement of repetitive stroking action was applied in order to prevent the heat build-up and the formation of grooves [76].

The polishing time was standardized to avoid bias. In the current study, the time of polishing with each instrument was stan- 
dardized at $30 \mathrm{sec}$ because the most relevant research used 20 - 30 sec per polisher [76]. To avoid bias in the results of the present study, all the polishing systems had been undertaken by using the same slow-speed dental handpiece during all phases of the polishing procedure at a maximum speed of 20,000 rpm [69].

After polishing, the specimens were cleaned in an ultrasonic bath at $35^{\circ} \mathrm{C}$ for 3 min to remove any debris then dried with absorbent paper and absolute ethanol (90\%) using a microbrush [15].

The composite specimens had their thickness measured in triplicates with a digital caliper (Absolute Digimatic, Mitutoyo, Tokyo, Japan). Specimens that varied more than $0.05 \mathrm{~mm}$ from the ideal thickness (3 mm) were discarded [44].

\section{Surface roughness measurements (Ra)}

The average surface roughness ( $\mathrm{Ra}$ ) was measured after specimens polishing four times each with a cut-off value of $0.8 \mathrm{~mm}$, a transverse length of $0.8 \mathrm{~mm}$ and a stylus speed of $0.1 \mathrm{~mm} / \mathrm{sec}$ near the center of each specimen using a profilometer (Mitutoyo SJ-201 Surf tests, Japan).

\section{Statistical analysis}

Numerical data were explored for normality by checking the distribution of data and using tests of normality (KolmogorovSmirnov and Shapiro-Wilk tests). Data showed normal (parametric) distribution. Data were presented as mean and standard deviation (SD) values.

Repeated measures Analysis of Variance (ANOVA) was used to study the effect of composite type, polishing system, tooth brush type, brushing and their interactions on mean Ra.

Bonferroni's post-hoc test was used for pair-wise comparisons when ANOVA test is significant. The significance level was set at $\mathrm{P}$ $\leq 0.05$. Statistical analysis was performed with IBM SPSS Version 23.0. Armonk, NY: IBM Corp Statistics for Windows.

\section{Results}

\section{Effect of composite type regardless of other variables}

Regardless of polishing system; Filtek Z350 XT showed statistically significantly lower mean Ra than Filtek Z250 (P-value $=0.008$, Effect size $=0.136$ ) (Table 3 and figure 6).

\begin{tabular}{|c|c|c|c|c|c|}
\hline \multicolumn{2}{|c|}{ Filtek Z350 XT } & \multicolumn{2}{|c|}{ Filtek Z250 XT } & \multirow{2}{*}{$\begin{array}{c}\text { P- } \\
\text { value }\end{array}$} & \multirow{2}{*}{$\begin{array}{c}\text { Effect size } \\
\text { (Partial eta } \\
\text { squared) }\end{array}$} \\
\hline Mean & SD & Mean & SD & & \\
\hline 0.7118 & 0.2039 & 0.8279 & 0.385 & $0.008^{*}$ & 0.136 \\
\hline
\end{tabular}

Table 3: The mean, standard deviation (SD) values and results of repeated measures ANOVA test for comparison between Ra of the two composite types regardless of other variables.

*: Significant at $\mathrm{P} \leq 0.05$.

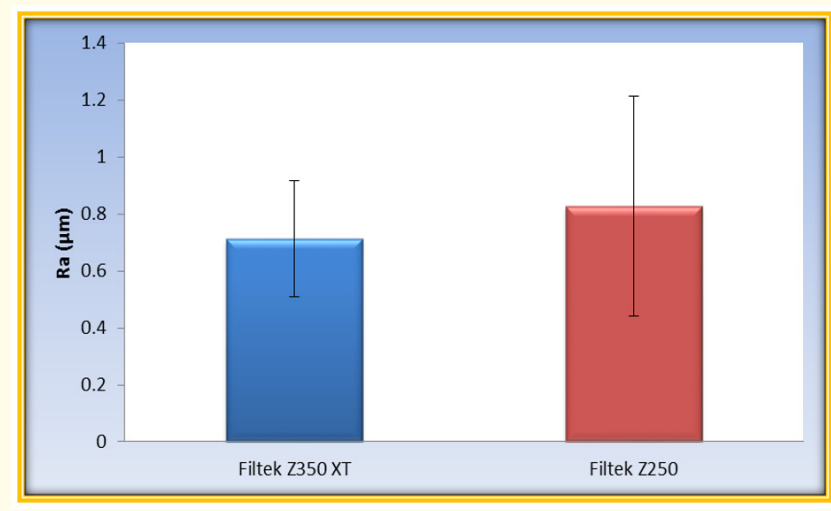

Figure 6: Bar chart representing mean and standard deviation values for Ra of the two composite types regardless of other variables.

Effect of polishing system regardless of other variables

Regardless of composite type, there was a statistically significant difference between mean Ra of different polishing systems (Pvalue $=0.001$, Effect size $=0.269$ ). Pair-wise comparisons revealed that, there was no statistically significant difference between PoGo discs and Sof-Lex discs; both showed statistically significantly higher mean Ra than Polyester strip (Table 4 and figure 7).

\section{Discussion}

In esthetic dentistry, restorative materials should duplicate the appearance of a natural tooth. A resin composite restoration can be unpredictable by the naked eye when its surface closely resembles the surrounding enamel surface. Thus, highly finished and polished restorations should produce an enamel-like surface texture and 


\begin{tabular}{|l|c|c|c|c|c|c|c|}
\hline \multicolumn{2}{|l|}{ PoGo } & \multicolumn{2}{|c|}{ Sof-Lex } & \multicolumn{2}{c|}{ Polyester strip } & \multirow{2}{*}{ P-value } & \multirow{2}{*}{ Effect size (Partial eta squared) } \\
\cline { 1 - 5 } Mean & SD & Mean & SD & Mean & SD & & \\
\hline $0.8026^{\mathrm{A}}$ & 0.309 & $0.8583^{\mathrm{A}}$ & 0.2406 & $0.6486^{\mathrm{B}}$ & 0.3464 & $0.001^{*}$ & 0.269 \\
\hline
\end{tabular}

Table 4: The mean, standard deviation (SD) values and results of repeated measures ANOVA test for comparison between Ra values of different polishing systems regardless of other variables

*: Significant at $\mathrm{P} \leq 0.05$, Different superscripts are statistically significantly different.

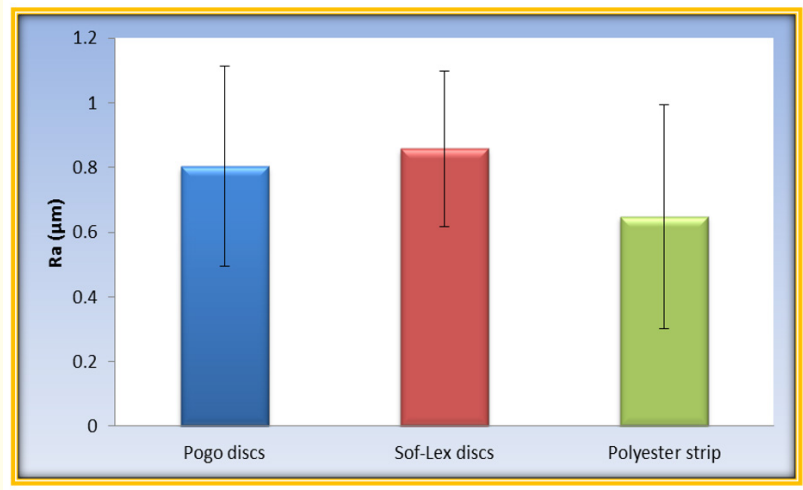

Figure 7: Bar chart representing mean and standard deviation values for Ra of different polishing systems regardless of other variables.

gloss. The clinical significance of surface roughness is related to decrease esthetic appearance of the restoration and discoloration. The biological consequences of the rough surface are the affection of gingival and periodontal health and the development of secondary caries due to increased plaque accumulation [27].

Therefore, the aim of the present study was to evaluate the surface roughness of two resin composites (Filtek Z350 XT nanofilled and Filtek Z250 XT nanohybrid) with different polishing techniques (PoGo single step and Sof-Lex multiple steps).

The commonly used nanofilled Filtek Z350 XT and nanohybrid Filtek Z250 XT resin composites were chosen in the present study because of the great advantages in their material compositions relating to the use of nanotechnology $[20,33]$. The application of nanotechnology to composite research has been of great benefit, due to reduced dimension of the particles and wider distribution; an increased filler load can be achieved, which results in high surface polish and better handling property $[12,27]$.

In the current study, a polyester matrix strip was used to produce standardized specimens. After photo-polymerization, the specimens that received no polishing served as controls and were compared with groups treated with different polishing systems. Such specimens, cured under matrix strips, have also been used as controls in several studies $[21,25]$. However, it is not always possible to use this method because of the anatomical complexity of the tooth [13].

Two polishing systems were used in the current study; PoGo single step polishers and Sof-Lex multiple steps contouring and polishing system because they reduce the clinical time and appear to be as effective as multi-step systems for finishing and polishing dental composites. The obvious advantage of the one-step system is the convenience and efficiency of producing a very smooth surface without having to switch to finer polishing items or having to wash and dry between each step to ensure removal of the larger abrasives from the previous step [5].

\section{Surface roughness}

Although a threshold for unacceptable surface roughness has not yet been agreed on, it was reported that, surface roughness above $0.2 \mu \mathrm{m}$ results in an increase of plaque accumulation, higher risk for caries and periodontal inflammation, compromising esthetics and longevity of the restoration [26].

However, [53] reported no difference in plaque accumulation throughout the roughness range of $0.7-1.4 \mu \mathrm{m}$. Thus, the clinical acceptable threshold level of the surface roughness in present study was situated at $8 \mu \mathrm{m}$. Since most treated surfaces presented 
surface roughness values in the range of $0.2-1.4 \mu \mathrm{m}$, the effect of the polishing systems on the finished surface of resin composites investigated is clinically relevant.

Generally, surface roughness of resin composites depends on several intrinsic and extrinsic factors. Intrinsic factors include: type of material, type of filler, shape, size and distribution of filler particles, degree of polymerization, resin matrix composition and durability of filler/matrix bond. Extrinsic factors are related to the method of polishing and include the flexibility of polishing tool, hardness of abrasive particles, geometrical shape and chemical structure of polishing tool, polishing time, applied force and its method of application $[36,46]$.

\section{Effect of composite type}

Filtek Z350 XT composite showed statistically significantly lower surface roughness $(0.71 \mu \mathrm{m})$ than Filtek Z250 XT composite $(0.82 \mu \mathrm{m})$ (Table 3 and figure 6$)$. Thus, the first null hypothesis that, there would be no difference in surface roughness values between nanofilled and nanohybrid composites was rejected.

This result could be attributed to different fillers' composition, size and loading of both tested materials. During the polishing procedure, in Filtek Z350 XT, nanomer and nanocluster particles were abraded easily along with the resin matrix. The nanomer bond which constructs nanoclusters would detach, providing a smoother surface. Also, nanomer was added with silane on its surface, which creates a strong bond with the matrix during curing. The matrix system contains more Bis-GMA and UDMA with less double bonds, increasing the degree of polymerization $[33,43]$.

While in Filtek Z250 XT resin composite, larger and irregular filler size was obtained by grinding larger particles and causing a lot of space between fillers. The larger filler would appear protrusive on the surface during curing. Pressure would gather more on the irregular filler and increase the chance of the filler detaching from the resin surface. When the larger filler detached from the matrix, it would create a large hole on the surface and increase surface roughness [33].

Additionally, Filtek Z250 XT resin composite still uses PEGDMA as a main matrix with more double bonds than Bis-GMA and UDMA, making the curing process less adequate than Filtek Z350 XT resin composite [33].
This result is in a wide agreement with those reported by $[14,20,42,60]$ who explained difference in surface roughness between Filtek Z350 XT and Filtek Z250 XT resin composites on the basis of differences in their chemical composition; nanofilled Filtek Z350 XT resin composite contains nanoparticles with an average size of $11 \mathrm{~nm}$ while nanohybrid Filtek Z250 XT resin composite has an average particle size of $0.6 \mu \mathrm{m}$.

On the other hand, this finding is in contrast with [53], who found that, Teric N-Ceram, which has been classified as nanohybrid composite as Z250 XT showed lower surface roughness compared to Filtek Z350 XT resin composite. This contradictory could be explained by difference in the brand of the nanohybrid composites used, which may have different fillers and matrix. Moreover, both studies have different methodologies; the surface of composite specimens had pre-finished with 1200 grit sand paper for $30 \mathrm{sec}$.

\section{Effect of polishing system}

There was no statistically significant difference between PoGo $(0.80 \mu \mathrm{m})$ and Sof-Lex $(0.85 \mu \mathrm{m})$ polishing systems, both showed statistically significantly higher surface roughness than polyester strip (0.64 $\mu \mathrm{m})$ (Table 3 and figure 6). Thus, the second hypothesis, that there would be no difference in surface roughness between the single step and multiple steps polishing systems was accepted.

These findings might be due to the polyester strip worked as a prohibition to format any oxygen inhibition layer over the surface of the uncured resin composite $[2,20,60]$.

These findings are in wide agreement with those reported by $[14,20,23,40]$ who found that, the smoothest surface obtainable on a composite restoration was that formed by a well-applied polyester strip, assuming the matrix was not allowed to move during the polymerization of the surface layer of the composite. The smooth surface formed by the matrix, which may include some imperfections, air inclusions and folds, tends to be rich in resin, but free of any air-inhibited composite. However, this surface has a resin-rich layer and presents a lower hardness. To prevent wear and discoloration, it is suggested to finish and polish this surface [36,55].

The similarity in the results of surface roughness for PoGo and Sof-Lex polishing systems were similar to those reported by [9,20], who found no difference between PoGo and Sof-Lex polishing sys- 
tems, which might be attributed to that, for a composite polishing system to be effective, the abrasive particles must be relatively harder than the fillers, if not, the polishing agent will only remove the soft resin matrix and leave the filler particles protruding from the surface [27].

Thus, it is believed that, the aluminum oxide abrasives in the Sof-Lex system and the diamond abrasives in the PoGo are higher in hardness than the most filler particles in resin composite used, which allow them to abrade the matrix and the filler particles equally promoting smooth surface for both resin restoratives $[2,20,53]$.

Contradictory results were obtained by [48], who found that, Sof-Lex spiral wheels created significantly smoother surfaces than PoGo micro-polisher. This may be explained by grounding the top surfaces of the composite discs with 600-grit silicon carbide paper for $20 \mathrm{sec}$.

Though there was no significant difference between the PoGo and Sof-Lex polishing systems the average mean roughness of PoGo system was lower than the Sof-Lex system, the excellent polishing ability of PoGo disc may be attributed to the fact that, the flexible PoGo disc contained fine diamond particles [20,53], which has harder diamond particles (7000 KHN) compared to aluminum oxide (2100 KHN) in Sof-Lex polishing system [27,51,53,55]. Moreover, a study by [58] demonstrated that, the Sof-Lex system created a more abrasive surface because it consists of three gradual files, starting from $98 \mu \mathrm{m}$ and progressing to $2 \mathrm{um}$ to $5 \mu \mathrm{m}$, to first contour and then polish the surface, moving from grit disc to another might cause scratches, grooves and cracks due to heat build-up resulting in more roughness $[9,42,65]$. Furthermore, [28] measured the temperature of dry polishing and found that, Sof-Lex spirals resulted in higher temperature than PoGo. The heat build-up can lead to the formation of grooves, which may result to higher surface roughness for Sof-Lex system [27].

On the other hand, the results of the current study are in disagreement with those obtained by [2], who found no significant difference between both polishing systems although the mean roughness values in PoGo micro-polisher were higher than the Sof-Lex polishing system. This might be due to variation in methodology, as the top surface of each sample was pre-roughened using 120-grit size sand paper.

\section{Conclusion}

Within the limitations of this study, the following conclusions can be drawn:

1. The nanofilled Filtek Z350 XT resin composite is a good choice material for the clinical use because it has better surface polishability.

2. The one-step PoGo micro-polisher produced better surface quality in terms of roughness than the multiple steps Sof-Lex polishing system.

\section{Bibliography}

1. Abed YA., et al. "Degree of conversion and surface hardness of bulk-fill composite versus incremental-fill composite". Tanta Dental Journal 12.2 (2015): 71-80.

2. Antonson SA., et al. "Comparison of different finishing/polishing systems on surface roughness and gloss of resin composites". Journal of Dentistry 39 (2011): e9-17.

3. Barakah HM and Taher NM. "Effect of polishing systems on stain susceptibility and surface roughness of nanocomposite resin material". Journal of Prosthetic Dentistry 112.3 (2014): 625-631.

4. Barbieri GM., et al. "Effect of whitening dentifrices on the surface roughness of commercial composites". Journal of Esthetic and Restorative Dentistry 23.5 (2011): 338-345.

5. Bashetty K and Joshi S. "The effect of one-step and multi-step polishing systems on surface texture of two different resin composites". Journal of Conservative Dentistry 13.1 (2010): 3438.

6. Berger SB., et al. "Surface roughness and staining susceptibility of composite resins after finishing and polishing". Journal of Esthetic and Restorative Dentistry 23.1 (2011): 34-43.

7. Boutros F, et al. "Effect of tooth brushing on the surface roughness of different resin composites". Cairo University, MSc (2005).

8. Buchgraber B., et al. "Surface roughness of one nanofill and one Silorane composite after polishing". Collegium Antropologicum 35.3 (2011): 879-883. 
9. Canto FMT., et al. "Topography and microhardness changes of nanofilled resin composite restorations submitted to different finishing and polishing systems and erosive challenge". Brazilian Research in Pediatric Dentistry and Integrated Clinic 20 (2020): e4812.

10. Carneiro PMA., et al. "Influence of finishing and polishing techniques and abrasion on transmittance and roughness of composite resins". Operative Dentistry 41.6 (2016): 634-641.'

11. Carvalho FG., et al. "Effect of chemical and mechanical degradation on surface roughness of three glass-ionomers and a nanofilled resin composite". Operative Dentistry 37.5 (2012): 509-517.

12. Chour RG., et al. "Comparative evaluation of effect of different polishing systems on surface roughness of composite resin: An in-vitro study". Journal of International Society of Preventive and Community Dentistry 6.2 (2016): S166-170.

13. Colombo M., et al. "Effect of different finishing/polishing procedures on surface roughness of Ormocer-based and different resin composites". Journal of Dental Research 15.6 (2018): 404410.

14. Da Costa GD., et al. "Effect of additional polishing methods on the physical surface properties of different nanocomposites: SEM and AFM study". Microscopy Research and Technique 81.12 (2018): 1467-1473.

15. Da Costa J., et al. "The effect of various dentifrices on surface roughness and gloss of resin composites". Journal of Dentistry 38 (2010): e123-128.

16. Da Costa J., et al. "The effect of different polishing systems on surface roughness and gloss of various resin composites". Journal of Esthetic and Restorative Dentistry 19.4 (2007): 214-224.

17. Da Costa JB., et al. "Comparison of two-step versus four-step composite finishing/polishing disc systems: evaluation of a new two-step composite polishing disc system". Operative Dentistry 36.2 (2011): 205-212.

18. Da Rosa GM., et al. "Effect of whitening dentifrices on the surface roughness of a nanohybrid composite resin". European Journal of Dentistry 10.2 (2016): 170-175.
19. Dabhi MV., et al. "Comparative evaluation of three different types of tooth brush on surface abrasion of enamel and nanohybrid composite: An in-vitro study". IOSR Journal of Dental and Medical Sciences 15.8 (2016): 122-127.

20. Daud A., et al. "A randomized controlled study on the use of finishing and polishing systems on different resin composites using 3D contact optical profilometry and scanning electron microscopy". Journal of Dentistry 71 (2018): 25-30.

21. Durães I., et al. "Effects of different polishing systems on the surface roughness of two ceromers". Brazilian Dental Science 19.2 (2016): 56-63.

22. Eden E., et al. "The effect of finishing and polishing systems on surface roughness, microhardness and microleakage of a nanohybrid composite". Journal of International Dental and Medical Research 5.3 (2012): 155-160.

23. Ehrmann E., et al. "Finishing and polishing effects of multiblade burs on the surface texture of 5 resin composites: microhardness and roughness testing". Restorative Dentistry and Endodontics 44.1 (2019): 1-12.

24. Elbishari H., et al. "Is deterioration of surface properties of resin composites affected by filler size?" International Journal of Dentistry (2020): 2875262.

25. Erdemir U., et al. "The effect of one-step and multi-step polishing systems on the surface roughness and microhardness of novel resin composites". European Journal of Dentistry 60.2 (2012): 198-205.

26. Ereifej NS., et al. "The effect of polishing technique on 3D surface roughness and gloss of dental restorative resin composites". Operative Dentistry 38.1 (2012): E9-20.

27. Ergücü $\mathrm{Z}$ and Türkün LS. "Surface roughness of novel resin composites polished with one-step systems". Operative Dentistry 32.2 (2007): 185-192.

28. Ertugrul IF., et al. "Effect of different dry-polishing regimens on the intrapulpal temperature assessed with pulpal blood microcirculation model". Journal of Esthetic and Restorative Dentistry 31.3 (2019): 268-274. 
29. Filho HN., et al. "Surface roughness of composite resins after finishing and polishing". Brazilian Dental Journal 14.1 (2003): 37-41.

30. Gönülol N and Yllmaz F. "The effects of finishing and polishing techniques on surface roughness and color stability of nanocomposites". Journal of Dentistry 40 (2012): e64-70.

31. Gupta S and Dhawan R. "The effect of various finishing and polishing systems on the surface roughness of four composite resin materials: An in-vitro study". International Journal of Contemporary Dental 3.2 (2012): 1-9.

32. Heintze S., et al. "Surface deterioration of dental materials after simulated tooth brushing in relation to brushing time and load". Dental Materials 26.4 (2010): 306-319.

33. Itanto BSH., et al. "Comparison of surface roughness of nanofilled and nanohybrid composite resins after polishing with a multi-step technique". Journal of Physics 884.1 (2017): 1-7.

34. Jin JIAN., et al. "Surface properties of universal and flowable nanohybrid composites after simulated tooth brushing". American Journal of Dentistry 27.3 (2014): 149-154.

35. Jung M., et al. "Surface geometry of four nanofiller and one hybrid composite after one-step and multiple-step polishing". Operative Dentistry 32.4 (2007): 347-355.

36. Kemaloglu H., et al. "Can reduced-step polishers be as effective as multiple-step polishers in enhancing surface smoothness?" Journal of Esthetic and Restorative Dentistry 29.1 (2017): 3140.

37. Korkmaz Y., et al. "The influence of one-step polishing systems on the surface roughness and microhardness of nanocomposites". Operative Dentistry 33.1 (2008): 44-50.

38. Kumar S., et al. "A profilometric study to assess the role of toothbrush and toothpaste in abrasion process". Journal of Dentistry 16.3 (2015): 267-273.

39. Kyoizumi H., et al. "Effects of toothbrush hardness on in vitro wear and roughness of composite resins". The Journal of Contemporary Dental Practice 14.6 (2013): 1137-1144.
40. Lassila L., et al. "Effects of different polishing protocols and curing time on surface properties of a bulk-fill composite resin". The Chinese Journal of Dental Research 23.1 (202): 63-69.

41. Lopes IAD., et al. "The effect of different finishing and polishing techniques on surface roughness and gloss of two nanocomposites". Saudi Dental Journal 30.3 (2018): 197-207.

42. Moda MD., et al. "Comparison of different polishing methods on the surface roughness of microhybrid, microfill, and nanofill composite resins". Journal of Investigative and Clinical Dentistry 9.1 (2018): e12287.

43. Monteiro B and Spohr AM. "Surface roughness of composite resins after simulated tooth brushing with different dentifrices". Journal of International Oral Health 7.7 (2015): 1-5.

44. Mozzaquatro LR., et al. "The effect of brushing and aging on the staining and smoothness of resin composites". Journal of Esthetic and Restorative Dentistry 29.2 (2017): E44-55.

45. Nagem-Filho H., et al. "Surface roughness of composite resins after finishing and polishing". Brazilian Dental Journal 14.1 (2003): 37-41.

46. Nasoohi N., et al. "Effects of wet and dry finishing and polishing on surface roughness and microhardness of composite resins". Journal of Dentistry 14.2 (2017): 69-75.

47. Nayyer M., et al. "Comparative abrasive wear resistance and surface analysis of dental resin-based materials". European Journal of Dentistry 12.1 (2018): 57-66.

48. Nithya K., et al. "Evaluation of surface roughness, hardness and gloss of composites after three different finishing and polishing techniques: An in-vitro study". The Cureus Journal of Medical Science 12.2 (2020): e7037.

49. Oliveira GUD., et al. "Impact of filler size and distribution on roughness and wear of composite resin after simulated tooth brushing". Journal of Applied Oral Science 20.5 (2012): 510-516.

50. O'Neill C., et al. "Effect of tooth brushing on gloss retention and surface roughness of five bulk-fill resin composites". Journal of Esthetic and Restorative Dentistry 30.1 (2018): 59-69. 
51. Ozel E., et al. "Effect of one-step polishing systems on surface roughness of different flowable restorative materials". Dental Materials Journal 27.6 (2008): 755-764.

52. Pala K., et al. "Evaluation of the surface hardness, roughness, gloss and color of composites after different finishing/polishing treatments and thermocycling using a multi-technique approach". Dental Materials Journal 35.2 (2016): 278-289.

53. Patel B., et al. "Effect of different polishing systems on the surface roughness of nano-hybrid composites". Journal of Conservative Dentistry 19.1 (2016): 37-40.

54. Penteado RA., et al. "Evaluation of surface roughness of microhybrid and nanofilled composites after pH-cycling and simulated tooth brushing". The Journal of Contemporary Dental Practice 11.6 (2010): E17-24.

55. Pierre L., et al. "Influence of polishing systems on surface roughness of composite resins: polishability of composite resins". Operative Dentistry 44.3 (2019): E122-132.

56. Prakki A., et al. "In-vitro wear, surface roughness and hardness of propanal-containing and diacetyl-containing novel composites and copolymers based on Bis-GMA analogs". Dental Materials 24.3 (2008): 410-417.

57. Rajan VV., et al. "Effects of tooth brushing on wear, surface roughness, and color stability of composite resins: A review". Journal of Pharmacy Research 12.1 (2018): 95-102.

58. Rigo LC., et al. "Influence of polishing system on the surface roughness of flowable and regular-viscosity bulk fill composites". International Symposium on Periodontics and Restorative Dentistry 38.4 (2018): e79-86.

59. Rocha ACD., et al. "Evaluation of surface roughness of a nanofill resin composite after simulated brushing and immersion in mouthrinses, alcohol and water". Materials Research 13.1 (2010): 77-80.

60. Rodrigues-Junior SA., et al. "Surface roughness and gloss of actual composites as polished with different polishing systems". Operative Dentistry 40.4 (2015): 418-429.
61. Roopa KB., et al. "Effect of whitening dentifrice on micro-hardness, color stability and surface roughness of aesthetic restorative materials". Journal of Clinical and Diagnostic Research 10.3 (2016): ZC06-11.

62. Roselino L., et al. "Color stability and surface roughness of composite resins submitted to brushing with bleaching toothpastes: An in-situ study". Journal of Esthetic and Restorative Dentistry 31.5 (2019): 486-492.

63. Roselino L., et al. "Effect of brushing time and dentifrice abrasiveness on color change and surface roughness of resin composites". Brazilian Dental Journal 26.5 (2015): 507-513.

64. Ruivo MA., et al. "Surface roughness and filler particles characterization of resin-based composites". Microscopy Research and Technique 82.10 (2019): 1756-1767.

65. Santos JH., et al. "Whitening toothpastes effect on nanoparticle resin composite roughness after a brushing challenge: An in vitro study". Journal of Clinical and Experimental Dentistry 11.4 (2019): e334-339.

66. Schmitt VL., et al. "Effect of the polishing procedures on color stability and surface roughness of composite resins". International Scholarly Research Notices (2011): 617672.

67. Senawongse P and Pongprueksa P. "Surface roughness of nanofill and nanohybrid resin composites after polishing and brushing". Journal of Esthetic and Restorative Dentistry 19.5 (2007): 265-275.

68. Shimokawa CAK., et al. "In-vitro evaluation of surface properties and wear resistance of conventional and bulk-fill resinbased composites after brushing with a dentifrice". Operative Dentistry 44.6 (2019): 637-647.

69. Silva FP., et al. "Surface topography, gloss and flexural strength of Pressable ceramic after finishing/polishing protocols". Brazilian Dental Journal 30.2 (2019): 164-170.

70. Soliman YA., et al. "Surface roughness of nanohybrid composites with different monomers after finishing and polishing with different polishing systems". Key Engineering Materials 835 (2020): 41-49. 
71. Somacal DC., et al. "Effect of pH cycling followed by simulated tooth brushing on the surface roughness and bacterial adhesion of bulk-fill composite resins". Operative Dentistry 45.2 (2020): 209-218.

72. Suzuki T., et al. "Toothbrush abrasion of resin composites with different filler concepts". World Journal of Dentistry 3.2 (2012): 184-193.

73. Suzuki T., et al. "Resistance of nanofill and nanohybrid resin composites to toothbrush abrasion with calcium carbonate slurry”. Dental Materials Journal 28.6 (2009): 708-716.

74. Tărăboanţă I., et al. "Roughness variation of a nanohybrid composite resin submitted to acid and abrasive challenges". Biomaterial and Medical Devices 24.2 (2020): 182-187.

75. Watanabe T., et al. "Influence of polishing instruments on the surface texture of resin composites". Quintessence International 37.1 (2007): 61-67.

76. Wheeler J., et al. "Evaluation of the effects of polishing systems on surface roughness and morphology of dental composite resin". British Dental Journal 228.7 (2020): 527-532.

77. Zairani O., et al. "The effect of toothbrush bristle stiffness on nanohybrid surface roughness". International Journal of Physics 884 (2017): 012008.

78. Zanatta FB., et al. "Biofilm removal and gingival abrasion with medium and soft toothbrushes". Oral Health and Preventive Dentistry 9.2 (2011): 177-183.

Volume 5 Issue 11 November 2021

(C) All rights are reserved by Nehad Wafik Basha., et al. 